\title{
Attentional and automatic processes in line tracing: Is tracing obligatory?
}

\author{
David Crundall \\ University of Nottingham, Nottingham, England \\ GeofF G. Cole \\ University of Durham, Durham, England \\ AND \\ GEOFFREY UNDERWOOD \\ University of Nottingham, Nottingham, England
}

\begin{abstract}
When comparing two target elements placed on the same convoluted curve, response times are dependent on the distance between the targets along the curve, despite being separated by a constant Euclidean distance. The present study assessed whether such line tracing is obligatory across the whole of the line even when the task demands do not require it, or whether it is an optional strategy that can be disregarded when the circumstances favor a different method of attentional deployment. Three experiments were conducted to assess whether attention can select only a portion of a curve to trace when it is strategically sensible to do so. The results suggest that attention can indeed jump over portions of a line that are irrelevant to task performance before tracing has begun. However, the final experiment suggests that line tracing may continue beyond the task-relevant portion of the line. We conclude that line tracing is a strategy whose initial deployment can be influenced by top-down factors, rather than an obligatory response triggered by the stimuli-although, once engaged, line tracing may be hard to stop.
\end{abstract}

When we are presented with a top-down plan of a maze, it might be assumed that we will traverse the pathways with our eyes, in a fashion similar to how we might explore the same maze on foot. Indeed, this appears to be the case. Crowe, Averbeck, Chafee, Anderson, and Georgopoulos (2000) asked participants to judge which exit would correctly lead out of a series of mazes. They found that response times (RTs) increased with the length of the path from the start point to the exit and were also related to the number of turns within the main path. Eye movements, consisting of a number of saccades and fixations, closely followed the main path, although the convolution of the path in between subsequent fixations was such that it led Crowe et al. to conclude that the main path is "mentally traversed" away from the point of fixation. Once covert attention reaches a certain point, a saccade is initiated to bring the focus of the eye in line with the focus of covert attention. At this point, covert attention moves away once again, traversing the maze until it needs to call another saccade.

These results argue for a covert, serial search of the maze. Similar conclusions were drawn by Jolicœur and colleagues (Jolicœur \& Ingleton, 1991; Jolicœur, Ullman, \& Mackay, 1986, 1991; McCormick \& Jolicœur, 1991, 1992, 1994). Their basic paradigm required participants to view two convoluted lines or curves, one of which would pass through the point of fixation. (We refer to all stimuli as lines, though it should be noted that most stimuli used in these experiments involve curved lines rather than straight lines.) The task was to say whether two targets were on the same line or on different lines, without moving the eyes. The first target was always at the point of fixation, and the second target could appear at one of eight locations arranged in a circle around the fixation point, so that all potential locations were the same distance in Euclidean space from the center. Four of the potential target locations were on the same line as the first target, and four were on the other line. When targets appeared on the same line - although the absolute distance between all combinations of targets was equalthe second target could increase in distance from the first target in regard to its relative position along the convoluted line. Jolicœur (Jolicœur et al., 1986, 1991) noted that RTs to targets on the same line varied as a function of the relative distance between the first and second target along the line. It appeared that when judging whether the two targets were on the same line, this required participants to move covert attention along the line. The further along the line that attention had to travel, the longer it took for participants to make the correct decision. These results were found even when the displays only appeared for a fraction of a second, thus ruling out eye movements as the main cause of the effect. 
To explain these results, Jolicœur (Jolicœur et al., 1986, 1991) proposed an attentional operator devoted to line tracing. McCormick and Jolicœur (1994) assessed three different models and concluded that a variant of the zoom lens model of attention (e.g., Eriksen \& St. James, 1986) accounted for the majority of the results (but see Houtkamp, Spekreijse, \& Roelfsema, 2003). For example, this model could explain why the proximity of a distractor line influenced the time it took to trace to a target. In order to avoid mistakes due to tracing the wrong line, Jolicœur et al. stipulated that the operator must only include the target line. Thus, the proximity of the distractor line constrains the size of the zoom lens operator, with closer distractor lines resulting in a smaller focal size for the operator slowing the tracing process.

Although the basic line-tracing phenomenon is widely accepted (if not the mechanism by which it is achieved; cf. Houtkamp et al., 2003), the relevance of the effect has been questioned. It has been argued that curvature and collinearity can be processed in parallel under certain conditions (Fahle, 1991; Field, Hayes, \& Hess, 1993; Kovács \& Julesz, 1993). Boucart, Delord, and Giersch (1994) argued that Jolicœur et al.'s $(1986,1991)$ tracing operator is simply too slow to provide any valuable input into the interpretation of natural scenes. They compared the serial process of the line tracing operator to the results of Biederman (1981), who found that a scene needed to be displayed for only $150 \mathrm{msec}$ for objects within that scene to be recognized from line drawings. Most theories of visual perception assume a rapid or instant preattentive process that initially parses a scene into candidate objects or proto-objects on the basis of Gestalt groupings of features (Baylis, 1998; Rensink, 2000; Treisman \& Gelade, 1980). In light of the fact that such perceptual processes may be preattentive, one might argue that the relatively slow linetracing operator of Jolicœur et al. is merely a curiosity that arises out of a specific paradigm, with little relevance to real-world perception.

There is, however, evidence to suggest that grouping or binding features together does require attention under some circumstances. For instance, Ben-Av, Sagi, and Braun (1992) found that grouping by Gestalt principles did not occur when attention was directed elsewhere. Roelfsema, Lamme, and Spekreijse (2000) have produced data that suggest that attention uses Gestalt criteria to spread across features in the visual scene. The act of segregating features from the background and then binding them together is, they argue, an attentive process that cannot be completed until attention has been paid to all of the features that are to be included in the object.

Scholte, Spekreijse, and Roelfsema (2001) suggested that there are two types of grouping. The first, base grouping, involves very fast grouping on the basis that certain neurons are tuned to certain feature configurations, be they specific orientations of bars or, for instance, specific faces. This process allows for the possibility of preattentive parsing of objects. The second process is termed incremental grouping. This process is activated if the first process fails to work, presumably because the object in question is atypical and involves a serial spread of attention across the features.
Although incremental grouping does provide a use for Jolicœur et al.'s $(1986,1991)$ line-tracing operator, there are differences between this operator and the incremental grouping process defined by Scholte et al. (2001). The incremental grouping process is defined as a spread of attention, where the result is that attention is paid to the whole contour or curve, resulting in perception of the curve as an object. The line-tracing operator of Jolicœur, however, moves along the line and does not explicitly leave a trail of attention in its wake. Houtkamp et al. (2003) have recently produced strong evidence that suggests that participants can still encode feature attributes on a line after Jolicœur's tracing operator would have passed over that location. This evidence lends support to the hypothesis of the spreading trace of attention, but see Crundall, Dewhurst, and Underwood (in press) for contrasting evidence.

A related and important issue concerning the tracing operator is the extent to which it is obligatory. In the present context, this refers to the strength of the link between a stimulus appearing and activation of the line-tracing operator. We do not suggest that every line that meets the retina must be traced, but any task-relevant stimulus that one attends to (endogenously or exogenously) may encourage line tracing even when it is not beneficial to the task. For instance, Avrahami (1999) has shown that attention will trace lines even when they are irrelevant to the task. Similarly, attention automatically spreads within the contour boundary of a simple line-defined figure if one part of it is cued (Egly, Driver, \& Rafal, 1994). Indeed, the central tenet of so-called object-based attention is that objects are constructed preattentively on the basis of, for instance, good continuation, proximity, and similarity. Additionally, many authors argue that when the visual input is parsed into figure and ground, all features of the resultant objects receive attention (e.g., Duncan, 1993; O’Craven, Downing, \& Kanwisher, 1999). Furthermore, Pringle and Egeth (1988) produced evidence to suggest that the process of line tracing is triggered by the stimuli, rather than being influenced by top-down factors. They tried to induce alternative strategies for processing two targets on a simple curve, but the line-tracing process remained resilient. Hence, the finding that the effect still occurs - even when alternative strategies would prove more efficient - suggests that the effect occurs as a result of bottom-up mechanisms. Finally, Lavie and Driver (1996) also reported data suggestive of line tracing despite its not being advantageous to the task. They presented participants displays containing two overlapping straight lines together with two target elements that would occur either within the same line or one on each of the two lines. The task was to indicate as quickly as possible whether the two targets were the same or different. Results showed that RTs were reduced for trials where targets appeared on the same line in comparison with when they appeared on different lines. Although this was interpreted as evidence for object-based attention at the time, more recent research has demonstrated that this object bias disappears when the overlapping straight lines are kinked. In fact, a between-object advantage can be found when the two between-object targets are collinear, but the within-object targets are not (Crundall, Cole, \& Galpin, 2007). This can be easily explained by a line-tracing opera- 
tor, since angles in lines take longer to negotiate than do straight lines. Rather than serving as conclusive evidence for object-based attention, Lavie and Driver's results may be due to inappropriate line tracing.

Most previous line-tracing studies require participants to trace from one target to another, where the first target is located at the point of fixation (but see Roelfsema, Khayat, \& Spekreijse, 2003, and Pringle \& Egeth, 1988, as noted exceptions). Participants therefore do not need to move attention to access the first target. Instead, they merely move attention along the line to find the second target. Thus, these studies are set up to test only the effects of attention between the two targets. Attention to those parts of the line outside that portion that falls between the two targets has not been studied. If line tracing is triggered by preattentive grouping processes concerned with parsing an object, participants should attend to the whole line; and as Pringle and Egeth found, this process would be obligatory and resistant to top-down control. The present study set out to examine whether line tracing would still occur were the first target not located at the central point of fixation, but the line on which both targets appeared still passed through the center of the display. Participants could quite easily complete the task by moving attention directly (without tracing) to one of the targets before tracing the line in the direction of the other target, whose rough location would be available extrafoveally.

However, if line tracing is an obligatory process, attention can be presumed to be spread throughout the whole of the object. This process may be triggered by the presence of the stimulus at the focus of attention, rather than by the desire to trace between two targets. Thus, since the line still passes through the point of fixation, tracing should begin at this point. The operator should then trace the line until the first target is reached, before tracing onward to reach the second target. In this situation, there is no task motivation for the tracing from fixation to the first target. If tracing is a strategy that can be influenced by top-down factors, then there should be no tracing effects between the point of fixation and the first target. If, however, tracing is a stimulus-triggered process, then - provided that attention is located at the fixation point in the first instancetracing effects should be noticed between the point of fixation and the first target.

\section{EXPERIMENT 1}

Experiment 1 is a straightforward replication of Jolicœur et al.'s (1986) basic study using two targets arranged on two convoluted lines. The two targets appear either on the same line or on different lines; the first target always appears at the center, whereas the second target appears at one of eight other locations. We predicted an increase in RTs as a function of the distance between the two targets that appear on the same line.

\section{Method}

Participants. Twenty undergraduates at the University of Nottingham took part. They had a mean age of 21 years, and 14 were female. All participants had normal or corrected-to-normal vision.
Stimuli and Apparatus. Each stimulus contained two nonintersecting, convoluted black lines and two filled, black circles - the targets, each subtending $0.4^{\circ}$ of visual angle - that lay on one or both of the lines. These features appeared on a white background. One of the targets would always appear at the center of the screen, through which one of the lines would always pass. If the second target appeared on the same line as the first target, it could do so at one of four locations along the convoluted line, with Location 1 being nearest to the target in the center of the screen and Location 4 being the farthest location along the line. Although these potential target locations differed in their distance to the central target along the convoluted line, the line was constructed so that each location was the same distance in Euclidean space from the central target. There were also four potential target locations for the second target on the other line, in relation to the first target. The first location on the second line was always diametrically opposed to the first target location on the same line as the first target, with subsequent target locations increasing in distance from the first target location along the line (see Figure 1). All eight targets were situated at intervals of $45^{\circ}$ around the midpoint of an invisible circle having a diameter of $10^{\circ}$.

Eight basic stimulus templates were generated, consisting of two convoluted lines - one of which passed through the point of fixation at the center of the display. Each template was used to create eight stimuli representing the eight configurations of the two targets. One target was always placed at the center of the image, where the participants had to fixate, and the second target could appear in any one of the eight potential locations. Each stimulus was then rotated $90^{\circ}$, $180^{\circ}$, and $270^{\circ}$, and horizontal and vertical mirror images were taken of the original template, creating six variants. This created a total number of 384 stimuli. A Pentium III PC was used with a 21-in. FD Trinitron CRT monitor. A chinrest was used to maintain the distance between the screen and the participant.

Design. A $2 \times 4$ within-groups design was used. The first factor was whether the two target elements appeared on the same line or on different lines. The second factor was the location of the second target in relation to the first. There were four locations where the target could appear, either on the same line as the first target or on a

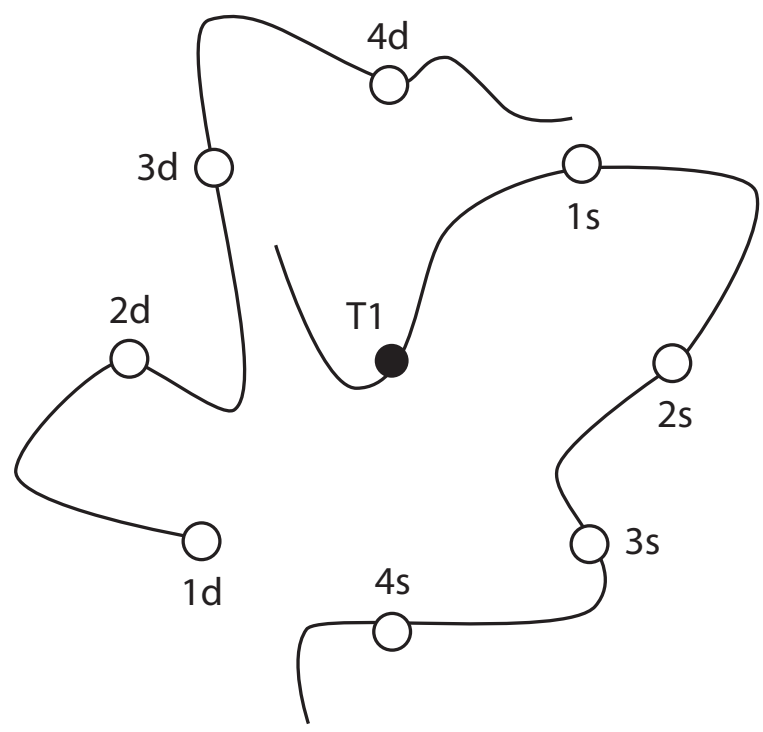

Figure 1.An example of two convoluted lines with the first target location marked (T1) and the eight potential target locations for the second target. Four of these target locations are on the same line as T1 (1s, $2 \mathrm{~s}, 3 \mathrm{~s}, 4 \mathrm{~s})$, and the other four target locations are on a different line (1d, 2d, 3d, 4d). The first target locations on each line (1s and 1d) were always diametrically opposed across T1. 
different line. When two targets appeared on the same line, the location of the second target determined the distance between the targets along the line. Participants had to respond according to whether the two targets appeared on the same line or on different lines. RTs were measured to test the hypothesis that the greater the distance between two targets, the longer it would take to compare the two.

Procedure. Participants were seated at a distance of $74 \mathrm{~cm}$ from the screen. Instructions were presented to the participants on the screen. Participants were informed that their task would require them to decide whether two targets were on the same line or on different lines. The numerical keys "1" and "2" were used for same and different responses, respectively. Participants could use either the numbers on the QWERTY keyboard or on the numerical keypad, depending on hand preference. Before the experiment began, participants were given a practice block of 16 trials taken at random from the 384 stimuli in the main experimental block. Before each display appeared, a black fixation cross (subtending $0.5^{\circ} \times 0.5^{\circ}$ ) was presented on a white background for $400 \mathrm{msec}$ at the center of the display. The stimulus was then presented for $250 \mathrm{msec}$, followed by a blank screen for $2,000 \mathrm{msec}$. If the participant failed to respond within this time period, this was considered an error, and the experiment proceeded to the following trial. Answering within the time limit resulted in auditory feedback.

\section{Results}

RTs were analyzed for outliers. One participant took over $2 S D$ s longer than the mean of the group in the majority of experimental conditions. This participant was removed from all further analyses, leaving 19 participants in the study. Incorrect responses and timed-out responses amounted to $5.8 \%$ of all responses.

A within-groups ANOVA compared RTs across the factors of response (same or different) and the position of the second target. A main effect was found for the type of response $\left[F(1,18)=29.4, M S_{\mathrm{e}}=7,251.6, p<.001\right]$, with same responses being faster than different responses. A main effect was also found for target location $[F(3,54)=$ 43.6, $\left.M S_{\mathrm{e}}=628.5, p<.001\right]$, and an interaction was noted between response and target location, even after the degrees of freedom were adjusted according to the Greenhouse-Geisser epsilon (to take into account problems with sphericity) $\left[F(2,36.3)=4.8, M S_{\mathrm{e}}=1,426.6\right.$, $p<.05$; see Figure $2 \mathrm{~A}]$. To further investigate the interaction, separate sets of planned contrasts were conducted on the RTs to targets on the same and different lines. For targets on the same line, the contrasts revealed differences between Locations 2 and $3\left[F(1,18)=17.5, M S_{\mathrm{e}}=977\right.$, $p<.001]$ and between Locations 3 and $4[F(1,18)=44.7$, $\left.M S_{\mathrm{e}}=1,349, p<.001\right]$. Contrasts performed on RTs to targets on different lines revealed a difference between Locations 3 and $4\left[F(1,18)=13.7, M S_{\mathrm{e}}=1,200, p<\right.$ $.005]$. In sum, these data show a curve-tracing effect. The speed with which observers can determine whether a peripheral item is on the same line as an item located at fixation increases as the distance along the line increases.

We additionally examined error rates for each condition in order to assess whether any form of speed-accuracy trade-off was operating. This analysis showed a similar pattern to RTs, with a significant interaction $[F(3,54)=$ $\left.10.7, M S_{\mathrm{e}}=15.6, p<.001\right]$ driven by a drop in accuracy with the longest line length between the two targets when they appear on the same line (Figure 2B). This rules out any possibility of a speed-accuracy trade-off.

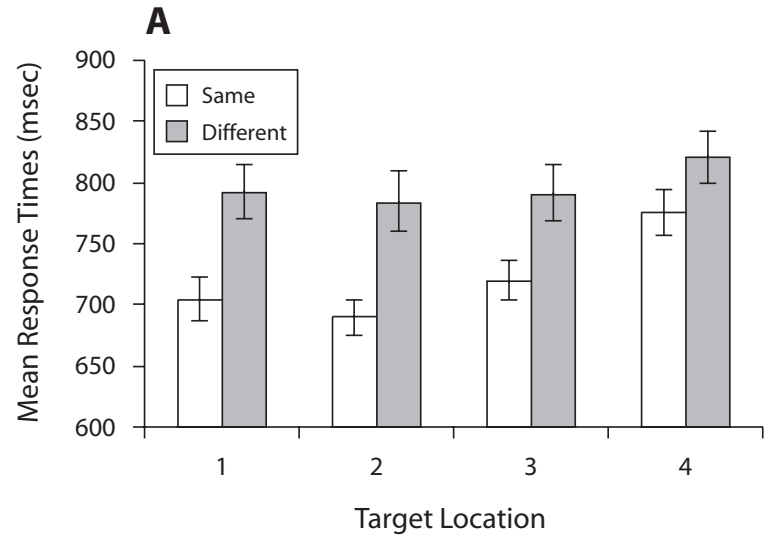

B

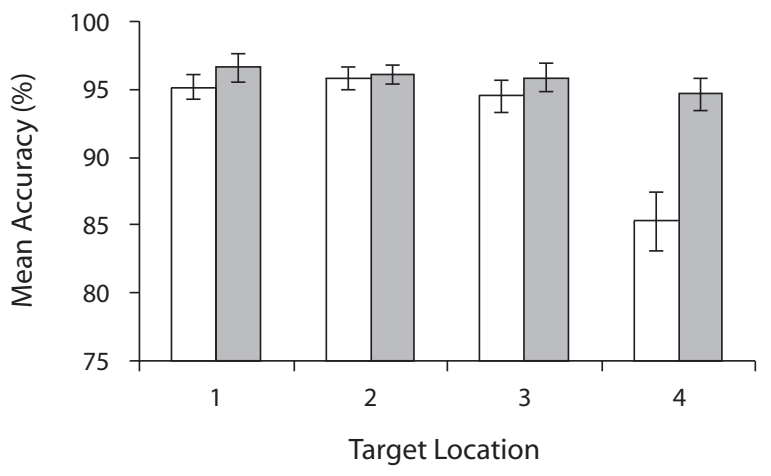

Figure 2. Experiment 1 mean response times (A) and mean percentage accuracy (B) to the appearance of two targets on either the same line or different lines, across four target locations (with standard error bars).

\section{Discussion}

The results are very similar to those reported by Jolicœur et al. (1986, Experiment 1). A general increase in RT is observed as the distance between two targets on the same line increases (with differences between Locations 2, 3, and 4 on the same line). According to Jolicœur et al. (1986), this provides evidence of a rapid internal tracing mechanism. The use of a $250-\mathrm{msec}$ stimulus duration also suggests that this effect is not dependent on eye movements. The present results failed to identify a difference between RTs to the two nearest target locations. Jolicœur et al. (1986) also reported the smallest differences between Locations 1 and 2, which perhaps suggests that tracing may not become evident until there is a relatively greater distance between the targets along the line. A further significant difference was noted between Locations 3 and 4 when the second target appeared on a different line from the first target. A similar trend can be seen in Jolicœur et al.'s (1986) data, with an increase of just under $40 \mathrm{msec}$ in RTs between Locations 2 and 3 . The present study found a difference of $29 \mathrm{msec}$ between Locations 3 and 4 to be significant.

If line tracing can only occur between targets on the same line, why should there be an increase in RTs due to locations on the different line? As Jolicœur et al. (1986) 
suggested, the participant may always trace the line on which the first target is located, with an increasing likelihood of giving a different response as the unsuccessful tracing of the first line progresses. This would explain why different responses are slower than same responses, but not necessarily why positions on the different line should produce differences in RTs. However, if one considers the possibility that, on a certain number of different trials, the participant may try to confirm that the target is indeed on a different line, then there is the possibility that they may also trace the second line. The starting point for tracing the second line should be as close to the end of the first line as possible, to reduce the need to move attention between the lines, since too great a jump of attention may risk landing on the line that has just been traced. Thus, participants should begin tracing the second line at the location where the first line finishes, which would mean that Locations $\mathrm{d} 1, \mathrm{~d} 2, \mathrm{~d} 3$, and $\mathrm{d} 4$ on the different line would be traced in that order. This would explain longer RTs for the fourth location than for the other locations. These data from the different responses contradict the findings of Pringle and Egeth (1988), who tended to find either no difference or an actual decrease in RT, with increasing distance between two targets in the different trials. They suggested that line tracing could not explain their results for the different trials and therefore argued for two parallel processes, with an alternative strategy providing faster responses for different trials. These data do not necessarily support the notion of two processes, since the results of different trials can be explained within a single line-tracing process.

\section{EXPERIMENT 2}

Overall, the results from Experiment 1 support the notion of line tracing between two targets on the same line and possibly suggest that tracing may even occur on the different line. As noted earlier, however, in nearly all of the previous work that has been undertaken on this hypothesized attentional operator, the first target has always appeared at fixation, and the second target has appeared extrafoveally. These stimuli presumably encourage line tracing, since it is inherent in the nature of the task - that is, checking that two targets are on the same line forces participants to trace an unpredictable line. Would participants still trace the line were it not beneficial to the outcome of the task, however?

In an attempt to test whether line tracing is obligatory or is, instead, a strategy that can be adopted according to task demands, we presented both targets displaced from the point of fixation, and one of the lines still passed through this point. In this situation, there are two portions of the line that can be traced when both targets are on the same line: from the point of fixation to the first target and from the first target to the second target. Experiment 2 thus sought to distinguish between an obligatory linetracing mechanism and a line-tracing strategy by varying the distance along the line between the point of fixation and the first target and by varying the distance between the two targets. Assuming that covert attention is coupled with the point of fixation prior to stimulus presentation, if line tracing is obligatory, attention should move away from the fixation point to the location of the first target, using the line as a guide for the attentional shift. Thus, RTs should increase with greater line length between the point of fixation and the first target along the line. If, however, line tracing is a strategy that can be applied only where appropriate, then attention should jump to the first target and only trace between the two. In this case, the distance between the fixation point and the first target along the line should have no effect on the RTs to both targets.

We incorporated further changes to Experiment 2 in order to control for a potential confound that might have arisen from a basic effect usually observed in tracing experiments. In Experiment 1, there was no difference in RT when the second target was in either Location 1 or Location 2. Jolicœur et al. (1986) found similar results in their original experiment. This may suggest that line tracing does not occur at short distances across the line, perhaps because the zoom lens operator can expand sufficiently to encompass the first target location without having to shift its focus. If this is the case, then a shift of attention would only be required when the target is in a more distant location along the line. For this reason, Target Locations 1s and 1d (see Figure 1) were not used in this experiment. Additionally, in order to ensure that target position would not confound the results, targets remained in the same place across conditions, and we varied line length between the point of fixation, Target 1, and Target 2 along the line instead of moving the target locations.

\section{Method}

Participants. Fifteen participants were recruited for Experiment 2. They had a mean age of 21 years, and 8 were female. All participants had normal or corrected-to-normal vision and had not taken part in the previous experiment.

Stimuli and Apparatus. The eight basic stimulus templates that were used in Experiment 1 were used in Experiment 2. Unlike Experiment 1 , no targets appeared at the center of the display. In half of the displays, the two targets appeared on the same line-again, always on the line that passed through fixation. Only four target locations were used in Experiment 2, equivalent to Locations $2 \mathrm{~s}, 4 \mathrm{~s}$, $2 \mathrm{~d}$, and $4 \mathrm{~d}$ from Experiment 1. When targets appeared on the same line, they would always appear in Locations $2 \mathrm{~s}$ and $4 \mathrm{~s}$ (a distance of $7^{\circ}$ ). Targets appearing on different lines appeared in all of the possible combinations an equal number of times.

The distance between the point of fixation and the first target (on the same line) and the distance between the first target and the second target were manipulated by increasing the convolution of the line. Figure 3 displays the four possible combinations of line convolution with one of the stimuli when two targets appeared on the same line. On different trials, the line that did not pass through the point of fixation was modified, with either the first half or the second half being increased in length and convolution. This manipulation was not intended to directly match the manipulation of line length in the same target trials, but merely to provide variation in both lines, so that participants did not notice a systematic difference in the extent of line convolution across the response condition. As in Experiment 1, targets were also equally distant from the point of fixation. Each basic template had four same response trials and four different response trials based on it (with the four same target trials representing the four combinations of line length between the point of fixation and the target and between the first target and the second target). The eight trials for each of the eight basic templates were also rotated through the four positions, as in the first experi- 
ment, and vertical and horizontal mirror images were taken from the original template. This created a total number of 384 trials. The apparatus was the same as that used in Experiment 1.

Design and Procedure. A $2 \times 2$ within-groups design was used, with the first factor as the distance from the point of fixation to the first target on the same line (either near or far) and the second as the distance between the two targets on the same line. Distances refers to the distances along the lines, rather than in Euclidean space, which was kept constant across the same target conditions. Different trials were not included in the subsequent analysis because the design of the experiment made it impossible to sensibly create valid conditions in the different target conditions. Different target responses had to be retained, however, for the task. The procedure was exactly the same as that for Experiment 1.

\section{Results}

The error rates for Experiment 2 were relatively high at $11.8 \%$. This proved to be due to 2 outlying participants who only achieved $52 \%$ and $59 \%$ accuracy, respectively. Since these participants were scoring close to chance expectancy, they were removed from further analysis. This reduced the overall error rate to a more consistent $6.8 \%$. The remaining RT data from 13 participants were subjected to a $2 \times 2$ ANOVA, with distance from fixation to Target 1 as the first variable and distance from Target 1 to Target 2 as the second variable. Responses to targets on different lines were not included. The results of the
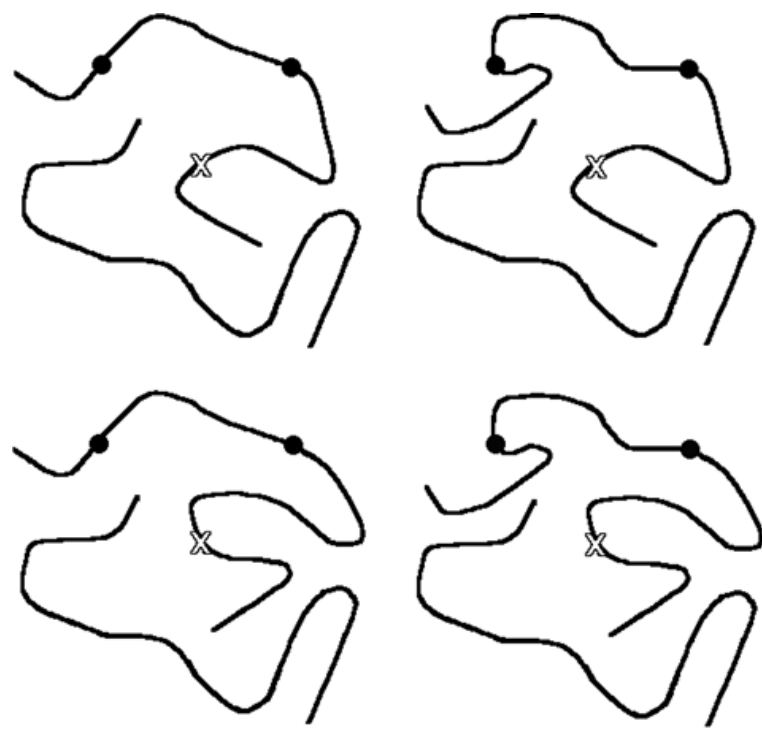

Figure 3. Four examples of stimuli used in Experiment 2 with targets on the same line. The top left panel contains a short distance along the line from the point of fixation to the first target and a short distance along the line from the first target to the second target. The top right panel contains a short distance between the fixation point and the first target and a long distance between the first target and the second target. The bottom left panel contains a long distance between the point of fixation and the first target and a short distance between the two targets. The bottom right panel contains long distances between the point of fixation and the first target and between the first and second targets. The white cross represents the location of the fixation cross prior to stimulus presentation. The fixation cross did not actually appear at the same time as the lines and targets.

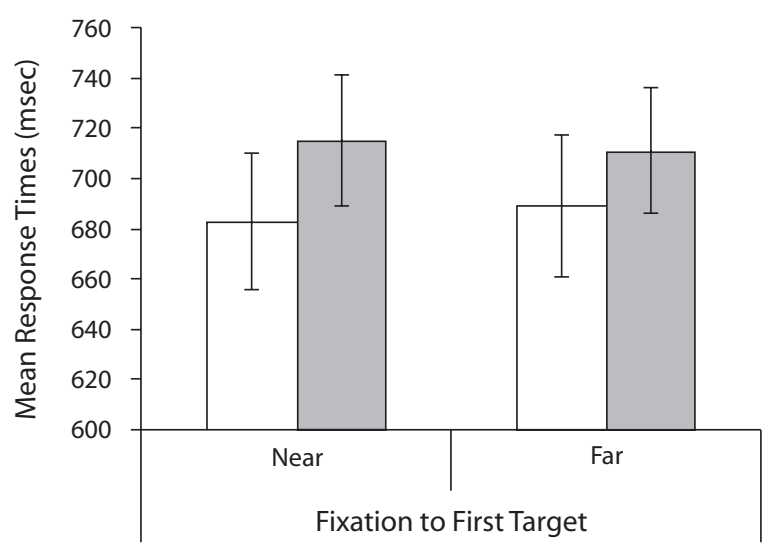

Figure 4. Experiment 2 mean response times (RTs) to the appearance of two targets on the same line, as a function of the line length between the point of fixation and the first target (near or far) and of the line length between the first target and the second target. Near RTs are depicted as empty bars; filled bars represent the far distance. Standard error bars are added.

ANOVA produced a main effect of the distance between the two targets on the same line $\left[F(1,12)=7.13, M S_{\mathrm{e}}=\right.$ $1,342.8, p<.05]$, with faster responses for short distances between Target 1 and Target 2. Varying the distance between the point of fixation and the first target did not have an effect on RTs $[F(1,12)=0.02]$, and the two factors did not interact. The means are displayed in Figure 4. These data clearly show that, when the task demands required it, tracing occurred between targets. By contrast, when tracing was not necessary, no tracing occurred. In other words, tracing is not obligatory in the sense that, although the participants' attention was required for moving from one point on the line (fixation) to another point on the same line (Target 1), participants did not use a curvetracing operator to make the shift, as Pringle and Egeth's (1988) results suggested.

As with Experiment 1, we again examined whether a speed-accuracy trade-off was operating by analyzing error rates for each condition. A $2 \times 2$ ANOVA on the percentage of correct responses mirrored the RT results, with an effect of line length between Targets 1 and 2, where short line lengths produced higher accuracy than long line lengths $[95 \%$ vs. $91 \%$, respectively; $F(1,12)=$ $\left.10.4, M S_{\mathrm{e}}=15.5, p<.01\right]$. The length of the line between fixation and Target 1 did not have an effect. These results suggest that no speed-accuracy trade-off was operating.

\section{Discussion}

The results demonstrated that extending the line between Target 1 and Target 2 had a detrimental effect on RTs. The manipulation of the line segment between the point of fixation and the first target did not, however, have any effect on RTs. These results suggest that, although there is evidence for line tracing between Targets 1 and 2, there is no evidence to suggest that line tracing occurs between the point of fixation and the first target. Instead, the alternative hypothesis - that attention may jump or move directly from the point of fixation to the first target - is supported. 
There is the possibility, however, that the difference between the near and far convolutions of the line between fixation and Target 1 were not extreme enough to produce noticeable tracing times, whereas the manipulation of the line length between the two targets obviously was. A third experiment was considered necessary to demonstrate that the manipulation of line length between fixation and Target 1 would produce RT differences under normal tracing conditions with a target at fixation, as in Experiment 1. This also provided an opportunity to test whether line tracing stops at Target 2 (i.e., does not trace beyond the necessary point on the line).

\section{EXPERIMENT 3}

Experiment 3 used all the same line stimuli that were used in Experiment 2, with all configurations of short and long variations. However, Target 1 always appeared at fixation, and Target 2 always appeared at Location 2 (although half the time this was on the distractor curve). The aim of this experiment was twofold. First, the variation of the line length between Targets 1 and 2 (the same variation as between the point of fixation and Target 1 in Experiment 2) was intended to demonstrate that the near and far conditions would produce different RTs if the portion of the line fell between the two targets rather than between the fixation point and Target 1 . Second, the variation of the line length after Target 2 (previously between Targets 1 and 2 in Experiment 2) should have no effect on RTs. This portion of the line is no longer relevant, perhaps even less so than the portion of the line between fixation and Target 1 in Experiment 2. Thus, an interaction between the line length between Targets 1 and 2 and the line length after Target 2 should be observed, with a tracing effect found between Targets 1 and 2, and no effect of line length after Target 2 .

\section{Method}

Participants. Ten participants were used. All participants had normal or corrected-to-normal vision and had not taken part in either Experiment 1 or 2.

Stimuli and Apparatus. The eight basic stimulus templates that were used in Experiments 1 and 2 were used in Experiment 3. Target 1 always appeared at fixation, and Target 2 always appeared at Location 2. On half of the trials, Target 2 appeared on the same curve, but on the other half, it appeared on the distractor curve.

The experiment was driven by a Pentium PC linked to a 21-in. Eizo monitor. All other aspects of the stimuli and apparatus were as described for the previous experiments.

Design and Procedure. A $2 \times 2$ within-groups design was used, with the first factor being the length of the line between Target 1 (at fixation) and Target 2 . The second factor was the length of the line after Target 2. As with Experiment 2, half of the trials had Target 2 on a different line, but these trials were not included in the subsequent analysis. The procedure was exactly the same as that used in the two previous experiments.

\section{Results}

The error rates for Experiment 3 were again relatively high at $10.5 \%$; but since no individual fell more than $2 S D$ s away from the group mean, all participants were retained for the analysis. Their RTs were subjected to a $2 \times$
2 ANOVA with line length from Target 1 (fixation) to Target 2 as the first variable and line length after Target 2 as the second variable. Responses to targets on different lines were not included. The results of the ANOVA produced a main effect of the distance between the two targets on the same line $\left[F(1,9)=14.4, M S_{\mathrm{e}}=512, p<.005\right]$, with faster responses for short distances between Target 1 and Target 2. Varying the line length after the second target also produced a main effect $\left[F(1,9)=10.9, M S_{\mathrm{e}}=941\right.$, $p<.01]$. The two factors did not interact. The means are displayed in Figure 5. Analysis of errors revealed no effect of line length and no interaction between the two. This suggests that no speed-accuracy trade-off was operating.

In sum, the variation of line length between Target 1 at fixation and Target 2 produced the expected effect on RTs, with longer line lengths producing slower RTs, indicating a line-tracing process. In addition, the length of the line following Target 2 appeared to influence RTs, with longer lines having a degrading effect on RTs.

\section{Discussion}

There are two important findings to take from Experiment 3. First, it is clear that there is a strong effect of line length between Targets 1 and 2 on RTs $\left(\eta_{\mathrm{p}}^{2}=.62\right)$. This suggests that the lack of an effect of line length between fixation and Target 1 (Experiment 2) was not due to the near and far conditions being too similar to produce a tracing effect under typical conditions (i.e., where Target 1 is at fixation). The second effect was more surprising. RTs were influenced by the length of the line following Target $2\left(\eta_{\mathrm{p}}^{2}=.55\right)$. This portion of the line was completely irrelevant to the task and should have had no effect on RTs. The fact that greater line length following Target 2 increased RTs raises the possibility that the tracing procedure continues past Target 2 to the end of the line.

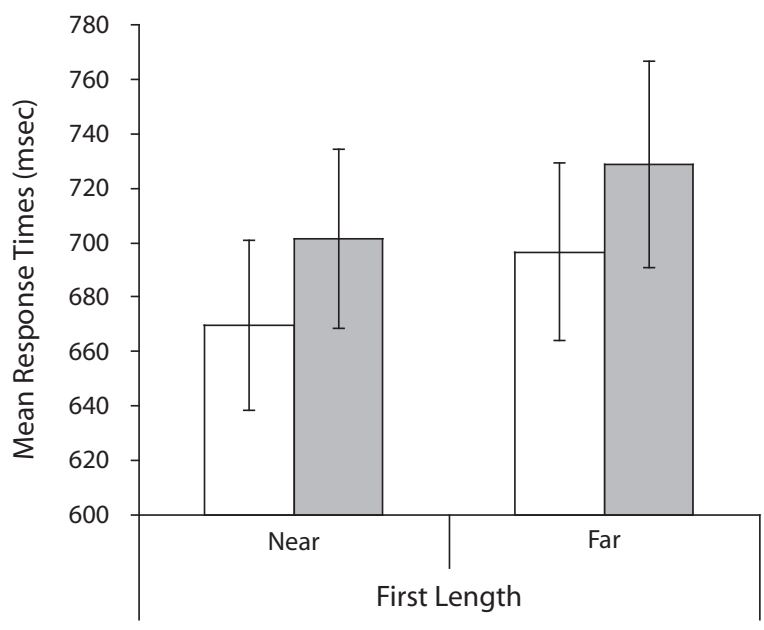

Figure 5. Mean response times (RTs) from Experiment 3 to the appearance of two targets on the same line, as a function of the line length between the two targets (near or far) and of the line length following the second target. Near RTs are depicted as empty bars; filled bars represent the far distance. Standard error bars are added. 


\section{GENERAL DISCUSSION}

To summarize, Experiment 1 replicated the line-tracing effect of Jolicœur et al. (1986), with slower RTs corresponding with an increase in line length between targets. Experiment 2 varied the line length between fixation and Target 1 and between Target 1 and Target 2. Only the latter had an impact on RTs, with greater line length producing slower responses. Experiment 3 demonstrated that the variation of line length between fixation and Target 1 in Experiment 2 could have a large effect on RTs, provided that that portion of the line lay between the two targets. Additionally, Experiment 3 suggested that the length of the line following Target 2 had an effect, with longer lines producing longer RTs, despite this portion of the lines being irrelevant to the task.

The results suggest that, under the appropriate conditions, line tracing is not obligatory. In Experiment 2, participants had no motivation to trace from the point of fixation to the location of the first target, and the evidence suggests that they did not. It appears that attention moved to the first target either in a direct analogue shift (see Tsal, 1983) or, perhaps, in a quantal jump (see Sperling \& Weichselgartner, 1995). On the basis of these results, we suggest that line tracing is not an obligatory process that is triggered by low-level features, but is, in fact, a strategy that can be influenced by top-down factors, which may determine when and where it would be best to deploy the tracing operator.

The fact that line tracing is not obligatory from the point of fixation under certain circumstances - and therefore does not always need to spread to the whole curve (or object) — does not necessarily rule out tracing as a mechanism for object parsing at the slower, incremental level. Other research on object-based attention has demonstrated that prioritization of certain stimuli can prevent object-based interference. Shomstein and Yantis (2002) looked for interference on responses to a central target letter from distracting flankers. These flankers could be contained within either the same rectangle as the target or a different, occluded rectangle. A strong version of the object-based attention theory would suggest that there is a greater chance of interference when the distractors are contained within the same rectangle, since an automatic spread of attention should process all parts of that object beforehand. In the first four experiments, they failed to find any influence of the rectangles on responses to the central target. In the final experiment, however, they introduced uncertainty about where the target would appear. This manipulation produced the object-based interference that they were looking for. They concluded that, since participants knew where the target was going to appear in the first four experiments, there was no reason to process the object containing the target and distractors; devoting attention to the rectangle could not improve performance any more than devoting all attention to the central target could. In the final experiment, attention needed to be deployed across all letters, creating a situation in which attention to the rectangle was advantageous. Shomstein and Yantis concluded that object parsing is not undertaken in all situations. In those situations in which processing an object will not benefit the task, there is a possibility that, under the right conditions (such as incremental grouping), spreading attention to the whole of a currently fixated object can be inhibited. This concurs with the present results, which have demonstrated that line tracing is not an obligatory process that is triggered merely by fixating an object or collection of features. Instead, it is a strategy that can be employed when it is beneficial to do so.

The results of Experiment 3, however, suggest an important caveat. It appears that the line length after Target 2 also influenced RTs. One possible explanation for this is that the amount of information in each stimulus might have an overall influence on RTs. In such cases, long line lengths would increase RTs simply because the greater detail in the display delays the response, and not necessarily because the participant has to trace the extra line length. Although the line length manipulations on the distractor line were not identically matched with those on the target line, this post hoc hypothesis would still predict that greater distractor line length should also be related to RTs. Accordingly, we conducted a $2 \times 2$ ANOVA on the correct RTs to trials in which the second target appeared on the distractor line (Experiment 3). Neither of the factors produced an effect, and there was no interaction. We cannot definitely conclude that overall stimulus complexity did not play a role in the effects noted in Experiment 3; there is certainly no evidence in favor of this hypothesis.

One other possibility is that the line tracer continued on to the end of the line after passing Target 2. Thus, the greater line lengths in the portion of the line beyond Target 2 increase RTs as the line-tracing operator continues to the end of the line. This suggests that there is an obligatory part of the line-tracing process, in that the operator must finish tracing the curve once it has started. If this is indeed the case, how did Jolicœur et al. (1986) find the line-tracing effect in the first place? Since their lines did not differ in length, one could argue that RTs should have been identical, regardless of target position, if attention were required to trace the whole line. However, as noted in Experiment 3, the effect of line length after Target 2 is weaker than the effect of line length between the two targets. This may reflect the possibility that the operator only continues along the line after Target 2 on a proportion of the trials or only continues for part of the way along the line before the participant can override the operator. Whatever the reason, this weaker effect would still allow the stronger tracing effect between targets to be found. This could be viewed as a form of attentional momentum, whereby the operator continues beyond Target 2 because the low level features (e.g., good continuation) encourage it to do so.

Alternatively, one might be concerned that the increased convolution of the portion of the line beyond Target 2 brings it back closer to the portion of the line between Target 1 (fixation) and Target 2 . If this is the case, then longer lines beyond Target 2 might interfere with the tracing between Target 1 and Target 2, since the operator would have to be reduced in size to avoid tracing the wrong portion of the line. A small number of our stimuli do indeed have 
such a feature, which might, at least in part, account for the post-Target 2 line convolution's influence on line tracing between Target 1 and Target 2 . This does not detract from the main conclusion, however. Whatever the reason for the influence of the line length beyond the target, the same influence should have been present in Experiment 2 had tracing occurred. The fact that it did not occur supports the primary finding, that the initial triggering of the line-tracing operator is under top-down control.

\section{AUTHOR NOTE}

This research was supported by U.K. Economic and Social Research Council Grant RES-000-22-0888. We thank Zia Hart for her help in stimuli design and data collection, Paul Skarratt also for assistance with data collection, and the participants for their time. Correspondence concerning this article should be addressed to D. Crundall, School of Psychology, University of Nottingham, University Park, Nottingham NG7 2RD, England (e-mail: david.crundall@nottingham.ac.uk).

\section{REFERENCES}

Avrahami, J. (1999). Objects of attention, objects of perception. Perception \& Psychophysics, 61, 1604-1612.

BAYLIS, G. C. (1998). Visual parsing and object-based attention: A developmental perspective. In J. E. Richards (Ed.), Cognitive neuroscience of attention: A developmental perspective (pp. 251-286). Mahwah, NJ: Erlbaum.

Ben-Av, M. B., SAGI, D., \& Braun, J. (1992). Visual attention and perceptual grouping. Perception \& Psychophysics, 52, 277-294.

Biederman, I. (1981). On the semantics of a glance at a scene. In M. Kubovy \& J. R. Pomerantz (Eds.), Perceptual organization (pp. 213-253). Hillsdale, NJ: Erlbaum.

Boucart, M., Delord, S., \& Giersch, A. (1994). The computation of contour information in complex objects. Perception, 23, 399-409.

Crowe, D. A., Averbeck, B. B., Chafee, M. V., Anderson, J. H., \& Georgopoulos, A. P. (2000). Mental maze solving. Journal of Cognitive Neuroscience, 12, 813-827.

Crundall, D., Cole, G. G., \& Galpin, A. (2007). Object-based attention is mediated by collinearity of targets. Quarterly Journal of Experimental Psychology, 60, 137-153.

Crundall, D., Dewhurst, R., \& Underwood, G. (in press). Does attention move or spread during mental curve tracing? Perception \& Psychophysics.

Duncan, J. (1993). Coordination of what and where in visual attention. Perception, 22, 1261-1270.

Egly, R., Driver, J., \& Rafal, R. D. (1994). Shifting visual attention between objects and locations: Evidence from normal and parietal lesion subjects. Journal of Experimental Psychology: General, 123, 161-177.

ERIKSEn, C. W., \& St. James, J. D. (1986). Visual attention within and around the field of focal attention: A zoom lens model. Perception \& Psychophysics, 40, 225-240.

FAHLE, M. (1991). Parallel perception of vernier offsets, curvature, and chevrons in humans. Vision Research, 31, 2149-2184.

Field, D. J., Hayes, A., \& Hess, R. F. (1993). Contour integration by the human visual system: Evidence for a local "association field." Vision Research, 33, 173-193.

Houtkamp, R., Spekreisse, H., \& Roelfsema, P. R. (2003). A gradual spread of attention during mental curve tracing. Perception \& Psychophysics, 65, 1136-1144.

JoLicEUR, P., \& INGLETON, M. (1991). Size invariance in curve tracing. Memory \& Cognition, 19, 21-36.

Joliceur, P., Ullman, S., \& Mackay, M. (1986). Curve tracing: A possible basic operation in the perception of spatial relations. Memory \& Cognition, 14, 129-140.

Joliceur, P., Ullman, S., \& Mackay, M. (1991). Visual curve tracing properties. Journal of Experimental Psychology: Human Perception \& Performance, 17, 997-1022.

Kovács, I., \& Julesz, B. (1993). A closed curve is much more than an incomplete one: Effect of closure in figure-ground segmentation. Proceedings of the National Academy of Sciences, 90, 7495-7497.

Lavie, N., \& Driver, J. (1996). On the spatial extent of attention in object-based visual selection. Perception \& Psychophysics, 58, 1238-1251.

McCormick, P. A., \& Joliceur, P. (1991). Predicting the shape of distance functions in curve tracing: Evidence for a zoom lens operator. Memory \& Cognition, 19, 469-486.

McCormick, P. A., \& Joliceur, P. (1992). Capturing visual attention and the curve tracing operation. Journal of Experimental Psychology: Human Perception \& Performance, 18, 72-89.

McCormick, P. A., \& Joliceur, P. (1994). Manipulating the shape of distance effects in visual curve tracing: Further evidence for the zoom lens model. Canadian Journal of Experimental Psychology, 48, 1-24.

O'Craven, K. M., Downing, P. E., \& Kanwisher, N. (1999). fMRI evidence for objects as the units of attentional selection. Nature, 401, 584-587.

Pringle, R., \& Egeth, H. E. (1988). Mental curve tracing with elementary stimuli. Journal of Experimental Psychology: Human Perception \& Performance, 14, 716-728.

Rensink, R. A. (2000). The dynamic representation of scenes. Visual Cognition, 7, 17-42.

Roelfsema, P. R., Khayat, P. S., \& Spekreijse, H. (2003). Subtask sequencing in the primary visual cortex. Proceedings of the National Academy of Sciences, 100, 5467-5472.

Roelfsema, P. R., Lamme, V. A. F., \& Spekreijse, H. (2000). The implementation of visual routines. Vision Research, 40, 1385-1411.

Scholte, H. S., Spekreisse, H., \& Roelfsema, P. R. (2001). The spatial profile of visual attention in mental curve tracing. Vision Research, 41, 2569-2580.

Shomstein, S., \& Yantis, S. (2002). Object-based attention: Sensory modulation or priority setting? Perception \& Psychophysics, 64, 41-51.

SPerling, G., \& Weichselgartner, E. (1995). Episodic theory of the dynamics of spatial attention. Psychological Review, 102, 503-532.

Treisman, A. M., \& Gelade, G. (1980). A feature-integration theory of attention. Cognitive Psychology, 12, 97-136.

Tsal, Y. (1983). Movement of attention across the visual field. Journal of Experimental Psychology: Human Perception \& Performance, 9, 523-530.

(Manuscript received June 1, 2006; revision accepted for publication September 20, 2007.) 